\title{
Experimental study on geomembrane expansion deformation under overlying water pressure and ring-restrained conditions
}

\author{
Ru-chun Wei ${ }^{1, a}$, Fang $\mathrm{Xu}^{1, \mathrm{~b}}$, Hong-fu Li ${ }^{2, \mathrm{c}}$, Wang-lin $\mathrm{Li}^{1, \mathrm{~d}, *}$, Zhan-lei Liu ${ }^{1, \mathrm{e}}$ and \\ Bo-fan Zhang ${ }^{1, f}$ \\ ${ }^{1}$ University of Jinan, Jinan 250002, China \\ ${ }^{2}$ Shandong Xin Hui Construction Group Co. Ltd., China \\ *Corresponding Author

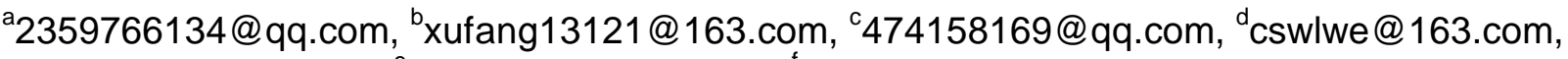 \\ eDick_heart@163.com, ${ }^{\mathrm{f}} 2195918394 @ q q . c o m$
}

Keywords: plain reservoir; geomembranes; overlying water pressure condition; expansion deformation; bursting strength

Abstract. The particular test apparatus was developed to measure the geomembrane expansion deformation under overlying water pressure and ring-restrained conditions. The geomembrane expansion deformation and failure experiment was performed and its rules were summarized. The conclusions are drawn as follows: (1) The factors that influence the geomembrane expansion deformation under overlying water pressure and ring-restrained conditions are apparatus aperture, loading rate and height of water column on the geomembrane. (2) Geomembrane bursting strength with the apparatus aperture of $100 \mathrm{~mm}$ is greater than the $200 \mathrm{~mm}$. (3) Bursting strength increases as the loading rate increases. (4) Height of water column on the geomembrane is proportional to the bursting strength.

\section{Introduction}

For the surrounding dam plain reservoirs that use a horizontal anti-seepage scheme, the geomembrane expansion deformation can be caused by factors such as the rise in groundwater table, the rapid reduction of reservoir water level, the filling surrounding dam and the leakage of geomembrane defect [1,2]. Considering facts such as the relative homogeneity of water and soil pressure on the geomembrane, the non-homogeneity of pore gas pressure under the geomembrane and the appearance of geomembrane expansion deformation, the geomembrane deformation is simplified as a spherical expansion deformation under a ring-restrained condition.

The characteristics of geomembrane expansion deformation are similar to the multi-axial tension deformation. Charalambides [3] obtained the strain value in the process of liquid expansion test by photogrammetric means, which solved the problem that it was difficulty in measuring the stress and strain of geomembranes. Bray [4] made assumption that the geomembrane expansion deformation was spherical and derived the relation between liquid expansion pressure and the geomembrane stress-strain by geometric methods, but this assumption would cause a large error of stress and strain obtained from the test due to the fact that the thickness and curvature of each point in the geomembrane deflection curve were not a constant under the hydraulic pressure. Yi-ming Shu [5] analyzed and summed up the relation between the liquid expansion ultimate load and the thickness of polyethylene geomembrane, the supporting layer and the aperture by using the theoretical simplification and tests. Wang-lin Li [6] presented relationship curves of geomembrane stress-strain and formulas of geomembrane stress and strain by testing geomembrane air expansion deformation under a ring-restrained condition. Nowadays, a number of relevant scholars have studied on the characteristics of geomembrane expansion and multi-axial tension deformation, but there are few researchers who performed tests on the mechanical properties of geomembrane expansion deformation under overlying water pressure and ring-restrained conditions.

Consequently, to study the mechanical properties of geomembrane expansion deformation under overlying pressure and ring-restrained conditions, a particular test apparatus that can be used to 
measure the geomembrane expansion deformation under overlying water pressure and ring-restrained conditions is developed to discover the deformation characteristics and rules, which is significantly important in ensuring the security and reliability of surrounding dam plain reservoirs that use a horizontal anti-seepage scheme.

\section{Test Design}

Test Apparatus. The particular test apparatus consists of seal system, water column applying load system, loading system and measurement and control system, as is shown in Fig. 1.

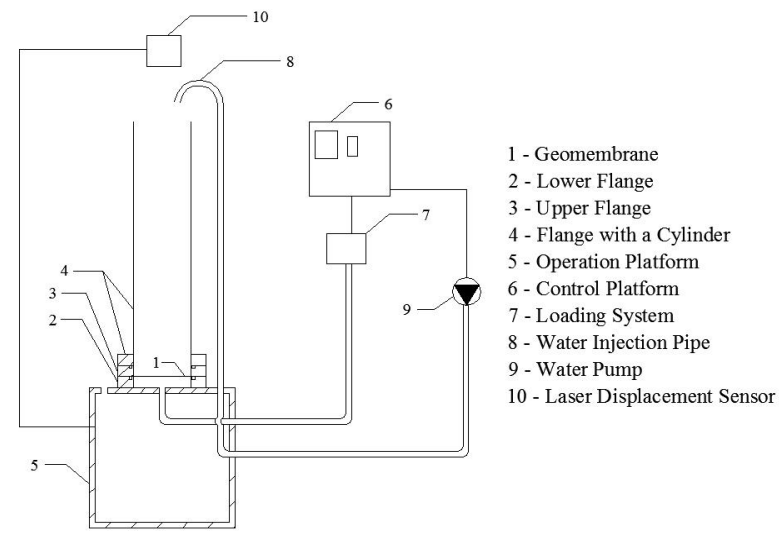

Fig.1 Schematic diagram of the test apparatus

The seal system consists of a couple of flanges and an operation platform, which is the fundamental platform of the test. The flanges that are machined from stainless steel with bolt holes including the lower flange and the upper flange is employed to clamp the geomembrane.

The water column applying load system consists of a stainless steel flange with an organic glass cylinder, a water injection pipe and a water pump. The water is injected into the cylinder by the water pump through the water injection pipe. The water height in the cylinder is used to simulate the pressure that is what the geomembrane bears in the actual projects.

The loading system is aimed at exerting a pressure under the geomembrane. Therefore, a special loading device that utilizes a motor driving a piston to exert pressure continuously for ensuring a sufficient and stable pressure supply during the test, which can be able to adjust the frequency of exerting pressure and be used repeatedly, is developed.

The measurement and control system consists of a precision digital pressure sensor, a ruler, a laser displacement sensor, a HD video recording system and a control platform. The crown height of geomembrane expansion deformation, the pressure in the container and the bursting strength (The failure pressure of geomembrane is called bursting strength.) of geomembranes can be measured and recorded automatically with the use of this system.

Test Material. The polyethylene geomembrane is chosen as test material. The sample thickness is $0.35 \mathrm{~mm}$ with diameters of $140 \mathrm{~mm}$ and $250 \mathrm{~mm}$ respectively. The geomembrane routine test index is shown in Table 1.

Table 1 Geomembrane routine test index

\begin{tabular}{cccc}
\hline Thickness $[\mathrm{mm}]$ & Mass per unit area $[\mathrm{g} / \mathrm{m} 2]$ & Tensile strength [MPa] & Elongation [\%] \\
\hline 0.35 & 455 & 20.74 & 644 \\
\hline
\end{tabular}

Test Scheme. In the test, the test apparatus utilized two different apparatus apertures (The apparatus aperture is the flange inner diameter.) that are $100 \mathrm{~mm}$ and $200 \mathrm{~mm}$ respectively. Six different loading rates were selected ranging from $6 \mathrm{~Hz}$ to $47 \mathrm{~Hz}$ respectively. And five different heights of water column were chosen such as $0 \mathrm{~mm}, 200 \mathrm{~mm}, 400 \mathrm{~mm}, 600 \mathrm{~mm}, 800 \mathrm{~mm}$.

\section{Test and Results}

The Effect of Apparatus Aperture and Loading Rate on the Bursting Strength of Geomembrane Expansion Deformation. Two different apparatus apertures of $100 \mathrm{~mm}$ and $200 \mathrm{~mm}$ were used for the 
test at six loading rates of $6 \mathrm{~Hz}, 8.4 \mathrm{~Hz}, 10.8 \mathrm{~Hz}, 13.2 \mathrm{~Hz}, 15.6 \mathrm{~Hz}$ and $18 \mathrm{~Hz}$ respectively. And the curve of geomembrane bursting strength with respect to the loading rate is described, as is shown in Fig. 2.

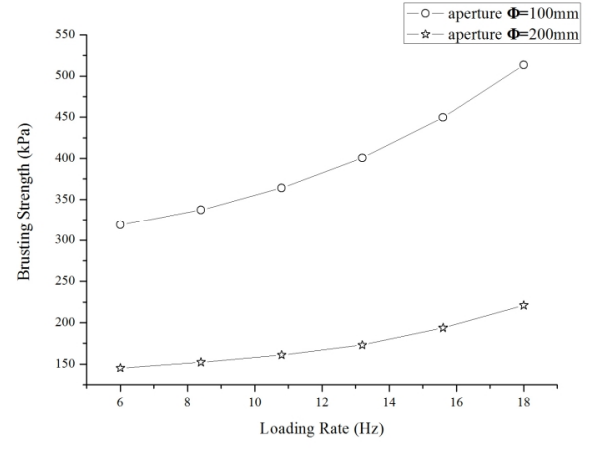

Fig. 2 Curve of the relation between the geomembrane bursting strength and loading rate in two apparatus apertures of $100 \mathrm{~mm}$ and $200 \mathrm{~mm}$ respectively

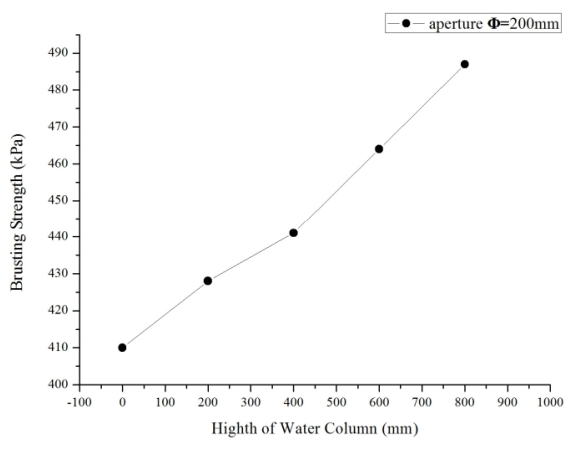

Fig.3 Curve of the relation between the bursting strength and height of water column on geomembrane in apparatus aperture of $200 \mathrm{~mm}$

As is shown in Fig. 2: an increase in a loading rate produces a bursting strength increase and at the same loading rate, the geomembrane bursting strength with respect to the apparatus aperture of $100 \mathrm{~mm}$ is greater than the $200 \mathrm{~mm}$. In addition, the bursting strength curve in $100 \mathrm{~mm}$ is slightly steep compared with the $200 \mathrm{~mm}$.

The Effect of Height of Water Column on the Bursting Strength of Geomembrane Expansion Deformation. Five heights of water column of $0 \mathrm{~mm}, 200 \mathrm{~mm}, 400 \mathrm{~mm}, 600 \mathrm{~mm}, 800 \mathrm{~mm}$ respectively with the apparatus aperture of $200 \mathrm{~mm}$ were used for test on the geomembrane. And the curve of geomembrane bursting strength with respect to the height of water column on the geomembrane is described, as is shown in Fig. 3.

As is shown in Fig. 3: the geomembrane bursting strength is nearly proportional to the height of water column on the geomembrane.

The Bursting Strength and Bursting Crown Height of Geomembrane Expansion Deformation. The crown height with geomembrane failure is called bursting crown height. Tests of geomembrane expansion deformation at different loading rates were performed with the apparatus aperture of $200 \mathrm{~mm}$ using the selected material, and conclusions are drawn as follows.

Under overlying water pressure and ring-restrained conditions, the bursting strength increases as the loading rate increases that the maximum is $623.8 \mathrm{kPa}$ at the loading rate of $47 \mathrm{~Hz}$ and is four times as many as the loading rate of $6 \mathrm{~Hz}$. At the same loading rate, the bursting strength increases as the height of water column increases and the bursting strength increment is equal to the pressure value which is applied on the geomembrane by the water column. The bursting crown height of geomembrane increases as the loading rate increases that the maximum is $86.1 \mathrm{~mm}$. Therefore, the water column on the geomembrane has no effect on the bursting crown height.

The Expansion Failure Forms and Characteristics of Geomembrane. The expansion failure form of geomembrane under overlying water pressure and ring-restrained conditions is similar to the air expansion failure, as is shown in Fig. 4.

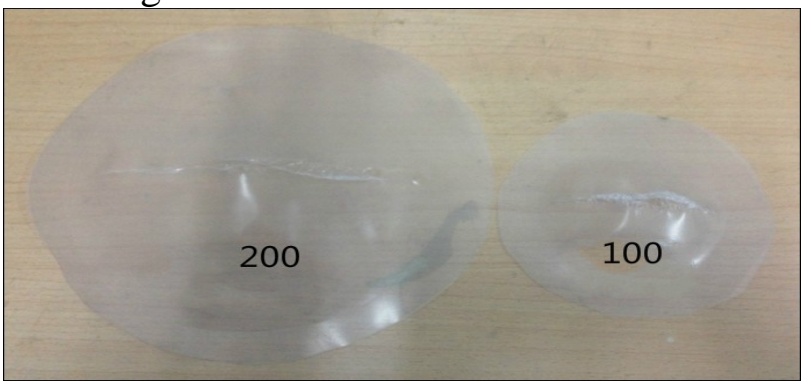

Fig.4 Typical expansion failure form for geomembrane sample

The expansion failure characteristics of geomembrane is as follows: 
The geomembrane expansion deformation is unhomogeneous due to the fact that the geomembrane thickness is unhomogeneous induced by expansion deformation. The geomembrane thickness is nonlinearly thinner gradually along a big circular curve from the restrained position of flange to the crown vertex.

In the process of the geomembrane expansion deformation, the tearing and tensile phenomenon begins to be produced when the stress reaches a certain value. This phenomenon is appeared firstly at a point near the top of the crown and then from this point, the trend continues to develop along a big circular curve or a small one until the geomembrane failure. The development of tearing direction is always along texture direction as a result of the geomembrane production line.

\section{Conclusions}

Conclusions are drawn as follows:

(1) The factors that influence the geomembrane expansion deformation under overlying water pressure and ring-restrained conditions are the apparatus aperture, the loading rate and the height of water column on the geomembrane.

(2) The geomembrane bursting strength with the apparatus aperture of $100 \mathrm{~mm}$ is greater than the $200 \mathrm{~mm}$. The bursting strength increases as the loading rate increases. The height of water column on the geomembrane is proportional to the bursting strength.

(3) The geomembrane expansion deformation is unhomogeneous and the expansion failure form is manifested as a big circular curve through the crown vertex or a small one not through the crown vertex.

\section{Acknowledgements}

This work was financially supported by Natural Science Foundation of Shandong Province (ZR2014EFM023), Shandong Province Science and Technology Development Plans (2013GSF11606) and Public Special Scientific Research of Ministry of Water Resources (201401024).

\section{References}

[1] Xue-shan Cao, Jun-ping Yuan, Gui-lin He, Ying-hao Liu and Zong-ze Yin: Geotextiles and Geomembranes. Vol. 43 No.1 (2015), p. 24-34.

[2] Wang-lin Li, Zhi-qiang Li, Xiao-yan Wei, Ying-te Li: Chinese Journal of Geotechnical Engineering. Vol.35 No.6 (2013), p. 1161-1165. In Chinese.

[3] M.N. Charalambides, L. Wanigasooriya, J.N. Williams and S. Chakrabarti: Rheol Acta, 2002, Vol. 41 p. $532-540$.

[4] J.D. Bray and S.M. Merry. Geosynthetics International, Vol.6 No.1 (1999), p. 19-40.

[5] Yi-ming Shu and Nai-hu Ye: Advances in Science and Technology of Water Resources. Vol. 23 No.5 (2013), p. 1-3. In Chinese.

[6] Wang-lin Li, Zhan-lei Liu, Xiang-tao Meng and Fang Xu: Chinese Journal of Geotechnical Engineering. Vol.38 No.6 (2016), p. 1147-1151. In Chinese. 